lpag

Business School

WORKING PAPER SERIES

Working Paper

2015-615
LE PLAN DE SAUVETAGE DE CHYPRE : FREIN OU ACCELERATEUR DU RISQUE SYSTEMIQUE EN EUROPE?

Jean Messiha

Frédéric Teulon

http://www.ipag.fr/fr/accueil/la-recherche/publications-WP.html

IPAG Business School

184, Boulevard Saint-Germain

75006 Paris

France 


\title{
LE PLAN DE SAUVETAGE DE CHYPRE : FREIN OU ACCELERATEUR DU RISQUE SYSTEMIQUE EN EUROPE ?
}

\author{
Jean Messiha \\ Administrateur civil hors classe \\ Economiste en chef \\ Ministère de la Défense - Délégation aux Affaires étrangères \\ jean.messiha@defense.gouv.fr \\ Frédéric Teulon \\ Département finance, IPAG Business School \\ Directeur de l'IPAG Research Lab \\ f.teulon@ipag.fr
}

\begin{abstract}
Résumé - Les institutions européennes ont manqué, sur le dossier chypriote, l'occasion d'asseoir leur crédibilité à bon compte. Non seulement, elles n'ont pas profité de la modestie du problème chypriote pour montrer comment elles pouvaient empêcher les risques systémiques de se développer dans la zone euro, mais elles ont de surcroît mené une action qui s'est avérée pro-cyclique. Ainsi, face à la dégradation de la situation à Chypre, l'Europe, de concert avec le FMI, a présenté un premier plan de sauvetage, dont l'une des mesures - la taxation de l'ensemble des dépôts - a eu pour effet d'aggraver la crise qu'elle souhaitait combattre.

Un nouveau plan plus équilibré a été conclu par l'Eurogroupe le 25 mars 2013. La taxation partielle des dépôts et l'adoption d'un contrôle des changes drastique constituent une violation du pacte de confiance qui liait jusqu'à présent les épargnants et leur banque. Chypre a été mise de fait sous tutelle économique.
\end{abstract}

\begin{abstract}
The European institutions have failed on the Cyprus issue, the opportunity to establish their credibility with it. Not only they have not benefited from the modesty of the Cyprus problem to show how they could prevent systemic risks grow in the euro area, but they additionally led an action proved pro-cyclical. Thus, faced with the deterioration of the situation in Cyprus, Europe, together with the IMF presented a first bailout, one action - the taxation of all deposits - has resulted aggravate the crisis she wanted to fight. A new plan was made more balanced by the Eurogroup on 25 March 2013. The partial taxation of deposits and the adoption of exchange controls drastically violate the pact of trust that binds far investors and their banks. Cyprus has been under de facto economic tutelage.
\end{abstract}

Classification JEL : E50, G15, G21.

Keywords : Chypre, Crise financière, Régulation de la zone euro.

Postprint : La Revue de la Régulation.

\footnotetext{
${ }^{1}$ Les opinions exprimées ici n'engagent que leurs auteurs et non les institutions auxquelles ils appartiennent.
} 


\section{Introduction}

L'économie chypriote a connu une croissance soutenue au cours des trois dernières décennies. L'activité a été tirée de l'avant par le tourisme et les services financiers et, dans une moindre mesure, par le secteur maritime (Devret, 2011). Le niveau de vie de la population s'est rapproché de la moyenne des pays de l'Union européenne (Tableau 1). L'intégration de cette île méditerranéenne dans l'Union européenne en 2004 a conforté ces tendances en dépit de la récession de 2009. Ainsi Chypre est devenue une zone à salaires élevés proche géographiquement de pays à coût de main-d'œuvre faible (Turquie, Liban, Egypte et Syrie). L'afflux de capitaux étrangers dont a bénéficié l'économie chypriote ne s'explique pas par la recherche d'une diversification géographique des placements ou par des coûts de production particulièrement bas; elle est surtout liée à des conditions fiscales jusqu'alors avantageuses et au statut offshore de l'île.

Tableau 1

Evolution du PIB/habitant

(base 100 - Europe des 27)

\begin{tabular}{lccc}
\hline & 1998 & 2003 & 2009 \\
\hline Chypre & 87 & 89 & 97 \\
Grèce & 83 & 92 & 95 \\
France & 115 & 112 & 107 \\
Turquie & 43 & 34 & 45 \\
\hline Source : Eurostat. & & &
\end{tabular}

Jusqu'à une date récente, les difficultés étaient surtout politiques et liées à la partition du territoire opérée en 1974, date de l'occupation du nord de l'île par la Turquie en réaction à une tentative de coup d'Etat qui aurait conduit à une union politique avec la Grèce (Eichengreen et al., 2004) : le gouvernement chypriote s'est retrouvé dans une situation comparable à la République fédérale d'Allemagne au moment de la signature du traité de Rome, il bénéficie de la reconnaissance internationale, mais il ne contrôle qu'une partie de son territoire.

Depuis la fin de l'année 2012, le pays est confronté à une crise financière de grande ampleur et il est entré dans une phase d'incertitude. Le paradis fiscal est devenu un pays proche de la faillite. Chypre a d'abord refusé de mettre en œuvre un premier plan de sauvetage (plan A), puis accepté un deuxième plan amendé (plan B) afin d'éviter d'être en situation de défaut de paiement et de sortir de la zone euro. Acculé, le gouvernement a finalement accepté de mettre en œuvre ce vaste plan de sauvetage dont les conditions ont été imposées de l'extérieur. La situation de Chypre met en évidence (comme cela a été le cas pour la Grèce) le poids des contraintes nationales dans le processus d'intégration européenne et les difficultés structurelles de régulation au sein de la zone euro. 


\section{Comment en est-on arrivé là ?}

Tant par sa taille que par son degré d'ouverture, l'économie chypriote est extrêmement intégrée à celle de ses voisins. De ce fait, la restructuration imposée aux créanciers privés de la Grèce au printemps 2012 s'est traduite par une brutale dévalorisation des titres de dette publique grecque détenus par les Chypriotes. Cet événement constitue le point de départ de la crise actuelle (Abid et al., 2013). La crise grecque a touché Chypre au travers de plusieurs canaux de transmission :

- le canal des finances publiques : les banques chypriotes affaiblies par les pertes sur les titres financiers grecs représentent désormais un poids pour le budget de l'Etat puisqu'il s'est avéré nécessaire de les renflouer ;

- le canal du crédit: la hausse des taux d'intérêt grecs engendrant un processus de défiance vis-à-vis de tous les pays périphériques ;

- le canal commercial : un peu plus de $1 / 5^{\text {ème }}$ des exportations de Chypre sont à destination de la Grèce ;

- le canal du prix des actifs : la chute des cours de la Bourse d'Athènes entrainant la chute de la Bourse de Nicosie. Les ménages détenteurs de titres financiers ont réduit leur consommation (effet de richesse).

Dans le processus d'effacement de 107 Mds EUR de dette souveraine grecque, Chypre a perdu 4,2 Mds EUR du fait des quantités importantes de titres de la dette publique grecque détenues par les banques chypriotes (soit près de $25 \%$ de la production de richesse annuelle, puisque le PIB de Chypre est de l'ordre de 18,5 Mds EUR). Si les pertes enregistrées sur la Grèce avaient été réparties au prorata de la richesse de chaque pays de la zone euro, Chypre aurait perdu seulement 200 millions d'euros... Bien évidemment une telle solution était difficilement envisageable car elle pose un problème d'aléa moral et parce qu'elle conduirait à absoudre les comportements des banques chypriotes qui ont spéculé sur le marché secondaire de la dette grecque. Toutefois, force est de constater que la résolution de la crise fait abstraction des critères politiques et géopolitiques qui forment pourtant le nœud du problème chypriote : absence de véritable solidarité budgétaire au sein de la zone euro ; admission au forceps de la Grèce dans l'euro alors que l'on savait dès les années 1980 que l'Etat grec ne parvenait pas à faire rentrer les impôts et qu'il avait engagé des réformes dispendieuses (Hibou, 1997) ; conflit helléno-turc qui fait de Chypre l'allié objectif de la Grèce (Bertrand, 2004); situation particulière des pays périphériques de l'Europe du Sud (Trimikliniotis, 2001).

Contrairement à une idée reçue, il ne s'agit donc pas initialement d'une crise d'endettement de l'Etat chypriote, puisque avant le défaut ordonné de la Grèce, Chypre affichait un ratio dette publique/PIB d'environ 60\% significativement inférieur aux ratios allemand et français (Tableau 2). La crise chypriote de la dette souveraine (16 Mds EUR de dette publique en 2012) peut donc être présentée comme un dommage collatéral provoqué par la crise grecque.

Tableau 2

Evolution de la dette publique de Chypre (en \% du PIB)

\begin{tabular}{crrrrr}
\hline 2007 & 2008 & 2009 & 2010 & 2011 & 2012 \\
\hline $58,8 \%$ & $58,9 \%$ & $58,5 \%$ & $62,3 \%$ & $71,1 \%$ & $85,8 \%$ \\
\hline
\end{tabular}


Chypre se trouve au cœur d'un des paradoxes européens: en privilégiant la stabilité monétaire (priorité absolue donnée à la lutte contre l'inflation) conformément aux enseignements de la théorie économique dominante (voir notamment Rogoff, 1985), la construction européenne favorise une instabilité financière qui risque de conduire certains Etats à la faillite.

Les crises grecques, irlandaises, portugaises ou chypriotes ont conduit la Banque centrale européenne (BCE) à mettre en œuvre des mesures inédites susceptibles d'ébranler les objectifs poursuivis (lutte contre l'inflation) et la crédibilité d'une institution supposée être la garante de la stabilité (Ugolini, 2011). Les Etats et les politiques publiques sont pris dans le « dilemme de la poule mouillée » mis en évidence par Sargent et Wallace (1981) : Comment rendre compatible une politique monétaire restrictive et une politique budgétaire laxiste ? Qui des autorités monétaires et budgétaires céderont le premier? De fait, la succession des crises contraint la $\mathrm{BCE}$ à mener une politique accommodante et l'offre de monnaie tend ainsi à devenir essentiellement endogène.

Notons que, dès le mois de juin 2012, une demande de renflouement du secteur bancaire de 17,5 Mds EUR avait été formulée par les autorités de l'île, requête jugée non urgente à l'époque par l'Eurogroupe. Depuis lors, la situation n'a cessé d'empirer au point de devenir financièrement insoutenable (on estime aujourd'hui les besoins financiers du pays à $23 \mathrm{Mds}$ EUR). Il est vrai qu'il était difficile de penser que l'économie chypriote, qui ne représente que $0,2 \%$ du PIB de la zone euro, puisse être à l'origine d'une crise systémique. En Europe, les banques, les investisseurs, les débiteurs et les Banques centrales sont liés par des chaînes multiples de financement et de refinancement croisés, par conséquent la défaillance de l'un d'entre eux peut à tout moment entraîner des pertes voire des défaillances pour les autres membres de la chaîne voire d'une autre chaîne qui la croise.

Quatre éléments se sont agrégés pour transformer le risque chypriote en risque global pour la zone euro :

- l'hypertrophie du secteur bancaire chypriote : alors que les actifs détenus par les banques est de 3,5 fois la valeur du PIB en moyenne dans la zone euro, ce coefficient est de 7,5 à Chypre (situation comparable à celle de Malte ou de l'Irlande, seul le Luxembourg - avec un ratio des actifs bancaires rapportés au PIB de 22 pour 1 - est dans une situation encore plus atypique);

- la surexposition du système financier chypriote au risque grec. L'« effacement volontaire " d'une partie de la dette souveraine grecque imposé aux créanciers conjuguée avec de nombreuses défaillances de la part d'emprunteurs helléniques privés ont conduit inéluctablement à une crise bancaire (Bourguignon, 2013) ;

- la gestion, mal adaptée par les institutions de l'UE, d'un problème circonstancié. Le plan de sauvetage conclu le 16 mars 2013 prévoyait notamment de taxer tous les dépôts bancaires à hauteur 9,9\% pour les dépôts supérieurs à 100.000 euros et $6,75 \%$ pour les dépôts inférieurs à cette somme. Cet impôt sur le capital devait dégager 5 à 6 Mds EUR et constituait la contrepartie du prêt de 10 Mds EUR que l'Union européenne (9 Mds EUR) et le FMI (1 Md EUR) étaient prêts à consentir à l'Etat Chypriote. Devant la colère populaire provoquée par cette taxe, la course aux guichets voire les risques d'insurrection qu'elle faisait courir au pays, le Parlement chypriote a refusé de voter le plan qui lui a été soumis le 19 mars. Le caractère draconien et inique $\mathrm{du}$ «plan $\mathrm{A} »$ (remise en cause du principe de garantie des petits dépôts) permet de se 
demander si l'on n'a pas voulu appliquer à Chypre une double peine : une peine au titre de maillon faible de la zone euro, puis une peine en tant que paradis fiscal ;

- Le choix de la méthode forte : le blocus monétaire organisé par la BCE a répondu au refus du plan de sauvetage par le Parlement chypriote. La BCE a en effet cessé d'alimenter la Banque centrale de Chypre en liquidités et a interrompu les transactions entre les banques chypriotes et le reste du système bancaire de la zone euro, excluant ainsi le pays du système européen des banques centrales (SEBC) et le plaçant de facto sous embargo.

\section{Où en est-on?}

Le plan de sauvetage prévoit que l'Union européenne et le FMI prêteront 10 Mds EUR à l'Etat chypriote qui apporte 13 Mds EUR provenant essentiellement de la taxation des dépôts supérieurs à 100000 euros (et uniquement de ceux-là). Toutefois, la taxation des dépôts supérieurs à 100000 euros ne devrait rapporter que 4,3 Mds EUR. Au vu de la fuite de capitaux massive, le taux nécessaire pour parvenir à dégager la somme recherchée pourrait avoisiner les $80 \%$.

\subsection{Les aspects conventionnels du plan de sauvetage de Chypre}

Le plan B, plus équilibré que le précédent, a été conclu par l'Eurogroupe le 25 mars 2013. En contrepartie des 10 Mds EUR, Chypre doit dégager 13 Mds EUR en procédant à deux réformes conventionnelles:

- une importante restructuration de son secteur bancaire. Il est prévu que Laïki Bank, la deuxième banque du pays dépose le bilan et qu'elle soit scindée en deux. Ainsi une structure de défaisance (bad bank) héberge les actifs toxiques et les dépôts nonsécurisés supérieurs à 100000 euros. Les prêts non risqués et les dépôts inférieurs à 100000 euros (garantis) sont placés au sein d'une structure particulière et saine. En renonçant à toute taxation des dépôts inférieurs à 100000 euros, ce nouveau plan met en place un prélèvement qui est assimilable à un impôt sur la fortune ;

- la création d'un Fonds spécial destiné à accueillir les revenus exceptionnels liés à la hausse des impôts, à la privatisation d'entreprises publiques et à la vente d'une partie de son stock d'or.

Tableau 3

Décomposition des sources de financement du plan de sauvetage de 23 Mds EUR

\begin{tabular}{|c|c|c|}
\hline & tien international & Mesures internes \\
\hline MES & $9 \mathrm{Mds}$ & $\begin{array}{l}\text { Liquidation et prélève- } \\
\text { ments bancaires } 10,6 \mathrm{Mds}\end{array}$ \\
\hline \multirow[t]{3}{*}{ FMI } & $1 \mathrm{Md}$ & Privatisations \\
\hline & & Hausse des impôts $0,6 \mathrm{Md}$ \\
\hline & & Ventes d'or \\
\hline
\end{tabular}


Au total, la contribution chypriote au plan de sauvetage proviendra pour l'essentiel $(10,6$ Mds EUR) de la liquidation de la Laiki Bank (vente d'actifs) et des ponctions effectuées sur les comptes de plus de 100000 euros. Le solde proviendra des hausses d'impôts, des privatisations et des ventes d'or (Tableau 3). Enfin un mémorandum de limitation des dépenses publiques complète le plan du 25 mars 2013.

\subsection{La naissance de facto d'un "euro chypriote"}

Au-delà de ces aspects, le plan du 25 mars prévoit une limitation des mouvements de capitaux. Cette mesure avait pour but d'éviter une trop grande pression sur les banques lors de leur réouverture quelques jours plus tard. Pour autant, la fuite des capitaux n'a pu être empêchée, puisque 4 à 10 Mds EUR seraient parvenus à quitter l'île, entre le 16 et le 25 mars, par le biais des filiales de la Laïki Bank et de la Bank of Cyprus, ainsi que par des clauses d'exceptions instaurées par la BCE afin de maîtriser les risques économiques afférents au blocus qu'elle organisait parallèlement.

La situation est pour le moins cocasse et paradoxale puisque l'on a instauré un contrôle des capitaux à l'intérieur d'une zone où une monnaie unique est supposée circuler librement. L'UE est contrainte de pousser Chypre à la non-application de l'un des principes sur lesquels elle s'est construite : l'absence de contrôle des changes. En violation de l'article 63 du TFUE, des limitations drastiques ont été établies par le gouvernement chypriote afin de limiter l'ampleur de l'hémorragie de capitaux. Cette mesure a provoqué deux effets contradictoires, l'un financier, l'autre réel :

- $\quad$ sur le plan financier, le risque sous-jacent d'une reprise immédiate de la fuite des capitaux a milité pour la prorogation d'un contrôle strict des mouvements de capitaux ;

- concomitamment et en termes réels, cette mesure a entraîné une exclusion de Chypre des transactions commerciales internationales (puisque importateurs et exportateurs nationaux ne peuvent ni payer ni être payés) ce qui a constitué une pression en faveur de sa levée rapide.

S'agissant de l'aspect strictement monétaire, l'isolement de la place financière de Chypre du reste de la zone euro a contribué de fait à différencier pendant un temps les euros circulant à Chypre de ceux circulant en dehors. Tout s'est passé comme si deux unités monétaires distinctes avaient coexisté, un "euro chypriote" et un "euro zone", le premier subissant une décote par rapport au second. Cette décote découle directement de la non-fongibilité temporaire entre "euro chypriote" et "euro zone", soit en réalité l'équivalent d'un taux de change fictif.

Cette mesure dont il ne faut pas sous-estimer la portée, nous renvoie à une réalité occultée depuis la signature du traité de Maastricht : le caractère supranational de l'euro rencontre des limites liée à la souveraineté des Etats qui gardent des moyens de pression sur la BCE et sur le pilotage de la masse monétaire en circulation dans la zone.

\subsection{Le rôle de la Russie}


La Russie s'est retrouvée en première ligne à Chypre du fait de l'ampleur des investissements réalisés par ses ressortissants : le montant des avoirs des banques et des sociétés russes dans les établissements financiers chypriotes dépasse les $25 \mathrm{Mds}$ EUR. Ces investissements obéissent à une logique fiscale, ils n'ont pas été réalisés dans le but de renforcer l'influence russe en Méditerranée. Cependant dans un contexte post Guerre froide, avec un gouvernement russe cherchant à rétablir son influence internationale (Melakopides, 2012), la crise chypriote place la Russie au premier plan.

Plusieurs questions se posent :

- sur le plan macroéconomique : il fut à un moment question que la Russie renfloue l'Etat chypriote contre des concessions gazières off shore qui auraient été accordées à la compagnie publique Gazprom. Qu'y a-t-il derrière cette proposition et pourquoi cela ne s'est-il pas fait ? Les Russes ont eu plusieurs mois pour négocier. Si un tel accord n'a pas été signé c'est qu'ils ne le souhaitaient pas ou parce qu'ils ont joué la carte de la dégradation de la situation afin d'être à terme dans une position plus favorable ;

- sur le plan microéconomique : il faut souligner le rôle des avoirs russes qui semblent avoir pesé sur l'attitude de l'UE et de l'Allemagne qui craignaient que ce soit in fine les gros comptes russes qui bénéficient de l'aide européenne et qui, de fait, ont décidé de remettre en cause le principe de sanctuarisation des comptes bancaires (comme les Russes représentent une grosse partie des dépôts, on peut avoir l'impression que taxer ces dépôts épargne les Européens).

\section{Où va-t-on ?}

\subsection{De nombreuses incertitudes}

Le projet initial de taxation des dépôts supérieurs à 100000 euros de la Bank of Cyprus ne devait rapporter que 4 Mds EUR. Au vu du montant des capitaux ayant déjà quitté l'île, le taux de taxation à appliquer à ces dépôts pour dégager la somme recherchée pourrait avoisiner le seuil très élevé et confiscatoire de $80 \%$. Si cette hypothèse se confirmait, le plan censé sauvegarder le secteur bancaire ne ferait que précipiter la chute de celui-ci. Les milliards manquants pourraient alors être trouvés au sein du fonds de solidarité dont la dotation proviendrait de l'exploitation future de gisements de gaz off shore situés en Mer Égée (dont la propriété est aussi revendiquée par la Grèce et la Turquie).

Les conditions assorties à la première intervention du MES (Mécanisme Européen de Solidarité) ont installé un climat de défiance dans l'ensemble de la zone euro. Deux événements d'une gravité exceptionnelle ont marqué la négociation de ce plan et sont de nature à créer un précédent qu'il sera difficile d'oublier :

- la volonté de taxer les dépôts, en violation directe des engagements solennels pris par la BCE jusqu'ici ;

- l'exclusion ponctuelle de Chypre du SEBC, isolant temporairement le pays.

Ce programme a eu pour effet d'installer un doute quant à l'intangibilité des deux principes fondamentaux régissant le service public de la banque que sont la garantie des encaisses monétaires de la population et la garantie de l'intégrité du système des paiements.

Plus globalement, les institutions européennes ont manqué l'occasion d'asseoir leur crédibilité à bon compte : le montant initial requis pour le sauvetage de Chypre, 17,5 Mds EUR, est une goutte d'eau que le MES aurait pu financer sur une partie, au demeurant modeste, de ses fonds propres. Si telle avait été la solution choisie, l'UE aurait non seulement 
colmaté définitivement le risque, mais surtout elle aurait initié un processus de diffusion rapide de la confiance en direction des autres marchés fragilisés par la crise. A contrario, avoir failli sur un problème aussi circonscrit aura contribué à instiller le doute. Il est dans la nature de la monnaie d'inspirer la confiance, mais celle-ci est difficile à cerner, comme pu le dire Georg Simmel (1907) : «La confiance est en dernier ressort improuvable. C'est un mélange de connaissance et de croyance. »

\subsection{Un climat peu propice au redressement}

Des craintes et des doutes persistent sur le caractère adéquat des mesures adoptées du fait de trois problèmes qui risquent de conjuguer leurs effets et d'affecter directement la France et la plupart des pays de l'UE :

- la crainte des déposants. Certes, la panique bancaire annoncée à Chypre le jour de réouverture des banques n'a pas eu lieu du fait de la garantie maintenue sur les petits dépôts et parce que les retraits supérieurs à 300 euros restaient interdits. Mais la fuite des capitaux par voie électronique pendant la période de fermeture des banques en dit long sur les velléités de "bank run" de la population, chypriote et européenne. Le risque de course aux guichets peut désormais resurgir - qu'il soit justifié ou non - dès que sera évoquée l'hypothèse d'une aide financière de l'UE à un Etat de la zone euro. Par ailleurs, le prélèvement opéré sur les dépôts constitue un précédent susceptible d'inciter les ménages à placer leurs avoirs dans plusieurs banques de façon à ne jamais dépasser le seuil des 100000 euros. Les réactions face aux difficultés de la Grèce à honorer sa dette, ainsi que celles de l'Italie confrontée à une grave contraction du crédit, nous apporteront à l'avenir des éléments plus concrets sur l'état d'esprit des déposants;

- la crainte des investisseurs. La solution retenue consistant à taxer les dépôts bancaires supérieurs à 100000 euros, ainsi que les déclarations à la presse du président de l'Eurogroupe, Jeroen Dijsselbloem, selon lesquelles la taxe chypriote pourrait être étendue à d'autres pays en difficulté, sont de nature à fragiliser la sédentarité des capitaux au sein de la zone euro, déjà affectée par un vaste mouvement de migration de l'épargne des pays du Sud vers ceux du Nord. La structure de l'épargne chypriote étant marquée par la place des capitaux venus du Moyen-Orient, il est par ailleurs possible qu'un volume important de capitaux aille se replacer en dehors de la zone euro, au profit de la zone dollar, de la livre sterling et/ou de l'or notamment.

- la crainte des entrepreneurs. L'actuelle paralysie de l'économie chypriote est propre à inspirer une généralisation de la méfiance des entrepreneurs à l'égard des pays en difficulté de la zone euro et, par conséquent, à stimuler à la fois une délocalisation de l'appareil de production de ces pays et un alourdissement de la facture du "risque pays" qu'ils présentent dans les négociations commerciales. Le plan actuel ne fait aucune différence entre personnes physiques et morales disposant de dépôts bancaires supérieurs à 100000 euros. La loi de restructuration bancaire votée le 22 mars, laisse toutefois à l'Etat chypriote la liberté d'assujettir ou non les personnes morales à la taxe sur les dépôts. Désastreuses pour l'économie réelle, cette extension offrirait l'avantage d'accroître le rendement financier : en clair, exclure les PME-PMI de la taxe, c'est réduire considérablement son produit. Les autorités chypriotes sous pression financière extrême pourraient bien choisir de sacrifier les capacités de production du pays. 


\section{Conclusion}

Le modèle économique sur lequel Chypre avait construit une certaine prospérité (forte attractivité du territoire national permettant l'accueil de touristes et de capitaux étrangers) est désormais remis en cause (Richard, 2013). Comment l'Europe a-t-elle pu envisager des mesures (plan A) susceptibles de provoquer une panique bancaire dans toute l'Europe du Sud ? Quant au plan B, de sérieux doutes subsistent quant à la productivité financière des décisions adoptées: le plan de sauvetage démantèle ce qui a assuré jusqu'à présent l'attractivité de Chypre. Le pays est entré dans une zone de turbulences : contraction du PIB liée à la restructuration du secteur bancaire, gonflement de la dette publique (85\% en 2012 et sans doute près de $110 \%$ en 2013) alimenté par les prêts de l'Europe et du FMI.

Quelle reconversion peut-on envisager pour l'économie de ce pays ? C'est peut-être la question la plus importante vis-à-vis de laquelle le plan de sauvetage n'apporte pas de réponses. En tout cas si ce pays souhaite rester une place financière offshore, la sortie de la zone euro semble pour l'instant exclue.

\section{Bibliographie}

Abid, I., Ftiti, Z. et Guesmi, K., (2013), « Greece's stock market integration into Southeast Europe», IPAG Working Papers Series.

Bertrand, G., (2004), Le conflit helléno-turc: la confrontation des deux nationalismes à l'aube du $X X I^{e}$ siècle, Paris, Maisonneuve \& Larose.

Bourguignon, F., (2013), « Crise chypriote : une cascade d'erreurs!», Les Echos, 4 avril.

Drevet, J.-F., (2011), « Chypre entre l'Europe et la Turquie, Karthala.

Eichengreen, B., Faini, R., Hagen, J. et Wyploz, C. (2004), Economic Aspects of the Annan plan for the solution of the Cyprus Problem, Rapport au gouvernement de Chypre.

Hibou, B., (1997), «La Grèce dans l'Europe: le révélateur budgétaire », Cahiers d'Etude sur la Méditerranée Orientale et le monde Turko-Iranien, vol. 23, p. 12-18.

Ioakimoglou, E., Arestis, P., Bilias, Y., Kalosinatos, P., (2011), The Competiveness of Cyprus economy after the adoption of euro, Nicosia, INEK-PEO.

Melakopides, C., (2012), « The 'Pragmatic Idealism' of Russia's Post-Cold War Policy towards Cyprus, », The Cyprus Review, vol. 24 (1), p. 71-97.

Richard, S., (2013), «La zone euro au révélateur chypriote », Question d'Europe, Policy Paper, $\mathrm{n}^{\circ} 273$.

Rogoff, K., (1985), « The Optimal Degree of Commitment to an Intermediate Monetary Target », Quarterly Journal of Economics, vol. 100 (4), p. 1169-1189.

Sargent, T.J., et Wallace, N., (1981), « Some Unpleasant Monetarist Arithmetic », Quarterly Review of Federal Reserve Bank of Minneapolis, vol. 5 (3), p. 1-17.

Simmel, G., (1907, 1 ère édition), Philosophie de l'argent, Paris, Presses Universitaires de France.

Trimikliniotis, N., (2001), "The Location of Cyprus in the Southern European Context: Europeanisation as Modernisation? », The Cyprus Review, vol. 13 (2), p. 47-73.

Ugolini, S., (2011), «What future for central banking ? Insights from the past », VoxEu.org. 
